\title{
Research of Space Positioning Method Based on Sound Field HBT Interference
}

\author{
Jing ZOU ${ }^{1}$, Lei NIE ${ }^{1}, \quad$ Mengran LIU ${ }^{1}$ and Chuankai JIANG ${ }^{1}$ \\ ${ }^{1}$ School of Mechanical Engineering, Hubei University of Technology,China
}

\begin{abstract}
Based on Hanbury Brown-Twiss (HBT) interference in the sound field, a space positioning method is presented to realize the long-distance and high-precision positioning of sound sources in media. Firstly, theoretical model of HBT interference positioning is established. Location of the sound source can be acquired by analyzing the correlation function of the output signals. Then, sound source localization under different signal-to-noise ratios (SNR) shows that by this method, the sound source can be accurately found with six sensors (two arrays) even the SNR is low to 0.04. Positioning experiment in air is carried out, and the experimental results show that the sound source can be accurately located at 42 meters, and the positioning error is low to 0.1 meters. Thus the validity and accuracy of the HBT interference space location principle is demonstrated. It provides new ideas for the research of long-range target location in sound propagation media (air, water, etc.).
\end{abstract}

\section{Introduction}

Sound is an important carrier of information dissemination. Acoustic wave is almost the only form of long-distance energy propagation in water which can be practically used in underwater positioning. Sound source localization based on sensor arrays is a research direction focus which has wide application prospects in autopilot, drones and submarine detection. Currently, positioning with microphone sensor arrays is an effective method of sound source localization. The sound signals are acquired by sensor arrays and then the relationship between the sound source and the array structure is analyzed to obtain the position information of the sound source[1,2].

Up to now, this kind of passive sound source localization mainly can be classified into three categories: time different of arrival (TDOA), high resolution spectrum estimation and beam forming [3,4]. The TDOA is one of the most commonly used positioning methods because of its relatively smaller computational complexity and easier implementation. The time difference from sound sources to different microphone are used to estimate the position of the sound source. However, the positioning accuracy is not satisfactory for long distance and low SNR $[5,6]$. The positioning method based on high-resolution spectral estimation mainly comes from modern high-resolution spectrum estimation techniques. Due to the short-term and stability of sound signals, this method is difficult to meet the high resolution requirements . Beam forming method performs weighted summation and beam forming on the signals collected by each element and then find sound source location by guiding beams. But a large amount of calculation makes real-time positioning can hardly realized by beam forming method [7].

This paper proposes a space location method based on the sound field Hanbury Brown-Twiss (HBT) interference. This method can achieve long-range accurate positioning with low-frequency signals and low signal-to-noise ratio (SNR), and the limitation of sensor position in the array is not strict.

\section{Positioning principle}

It is well known that HBT interference experiment of light describes the correlation of the optical field intensity. When the time delay of the two light signals is zero, the correlation function is the maximum. As the time delay increases, the correlation function gradually decreases and finally tends to a constant. Since the sound wave had the same propagation mode with the light wave, the HBT interference theory is also validated for sound field. The signal received by the sensor includes the acoustic signal of the sound source and environmental noise. Because the source signal has intrinsic coherence while noise has not, it is very convenient to recognize the source signal from the background noise. The product of the sound source signals received by the two sensors (that is, the HBT correlation function) will change with the phase difference. When the phase difference is zero, the correlation function reaches maximum, and the relativity is the greatest. As the phase difference becomes larger, the correlation function gradually decreases. Thus the location with the largest correlation function must be the sound source.

*Corresponding author: leinie@hbut.edu.cn 
Take a simple triangle array as an example to illustrate the principle of HBT interference location. As shown in Fig. 1, sensor array is composed of three elements, $M_{1}, M_{2}$, and $M_{3}$. The $S$ represents sound source. A coordinate system is established, the origin of which is $M_{2}$. As shown in Fig. 1, the rectangular area is where the sound source may appear, and the scanning interval is $L$. The HBT correlation function of corresponding position is calculated to determine the position of the sound source.

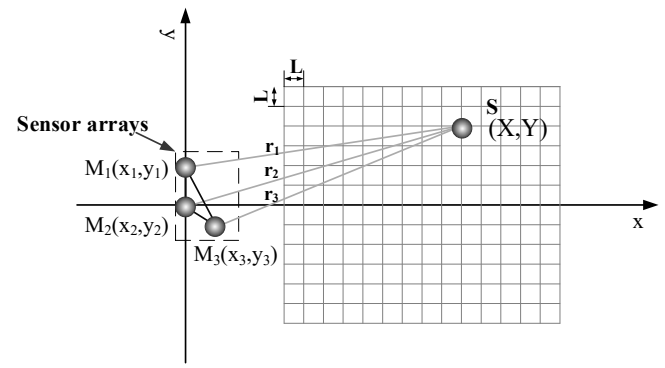

Figure 1. HBT interference positioning schematic

Sound waves travel in the form of spherical waves, thus, in this way, the signal $P_{i}$ received by the sensor can be represented as:

$$
P_{i}=N_{i}+\frac{A}{r_{i}} \cos \left[w\left(t-\frac{r_{i}}{v}\right)\right]+\varphi_{i}
$$

Where, $N_{i}$ represents the environmental noise, the A represents the amplitude of the sound source emission, $r_{i}$ indicates the distance between the sensor $M$ and the sound source. The $v$ represents the speed in media which is $1500 \mathrm{~m} / \mathrm{s}$ for the sound wave in water.

According to HBT interference principle,the normalized correlation functions $\mathrm{C}$ can be expressed as:

$$
\begin{aligned}
& C\left(\Delta T_{12}\right)=\frac{\left\langle P_{1}(t) \bullet P_{2}\left(t+\Delta T_{12}\right)\right\rangle}{\left\langle P_{1}(t)\right\rangle \bullet\left\langle P_{2}(t)\right\rangle} \\
& C\left(\Delta T_{13}\right)=\frac{\left\langle P_{1}(t) \bullet P_{3}\left(t+\Delta T_{13}\right)\right\rangle}{\left\langle P_{1}(t)\right\rangle \bullet\left\langle P_{3}(t)\right\rangle} \\
& C\left(\Delta T_{23}\right)=\frac{\left\langle P_{2}(t) \bullet P_{3}\left(t+\Delta T_{23}\right)\right\rangle}{\left\langle P_{2}(t)\right\rangle \bullet\left\langle P_{3}(t)\right\rangle},
\end{aligned}
$$

Where, $\Delta T$ represent time delay from sound source to sensor array.

The finalized normalized HBT correlation function is defined by:

$$
C=C\left(\Delta T_{12}\right) \times C\left(\Delta T_{13}\right) \times C\left(\Delta T_{23}\right) .
$$

\section{Simulation design}

In order to prove the validity of HBT interfere positioning method, two kinds of simulation, one is with different topologies and the other with different SNRs, are designed. The coordinate system of sensor arrays is shown in Fig. 1. The sound source is located at a distance of 5000 meters. According to practical environmental factors, the environmental noise is assumed as white noise. Since high-frequency sound signals attenuate much faster than low frequencies when traveling long distances, therefore, it is hypothesized that the frequency components of the sound source signal received by the sensor are $500 \mathrm{~Hz}$ and $315 \mathrm{~Hz}$, and the position coordinate of the preset sound source is $(5000$, 400), in meters.

Because the inter-array spacing is small, the angle between the sound source and the sensor element is small and the positioning resolution is reduced. Thus only the sound source direction can be determined with one array. Therefore there must be at least two arrays in long distance positioning. The intersection of two intersecting lines is the position of the source[8]. In practical environment, SNR is one of the most important research directions. The SNR is changed by changing the size of environment noise $N$ to achieve the research on different SNRs. This theory for different SNRs is analyzed in this paper.

\section{Result analysis}

\subsection{Sensor topology simulation analysis}

The position coordinates of the sensor array and corresponding simulation positioning results are shown in Table 1. Three kinds of topologies A, B, and C are two triangular arrays (array 1 and array 2) with spacing of 500, 800, and 1000 meters, respectively. As shown in Fig. 2(a), there are two triangular topological structures. Fig.2(b)-(d) shows the corresponding simulation results under different array topologies.

Table 1. Array coordinates and simulation result

\begin{tabular}{|c|c|c|c|}
\hline \multirow{2}{*}{ Array } & \multicolumn{2}{|c|}{ Array structure } & \multirow{2}{*}{$\begin{array}{c}\text { Simulation } \\
\text { results }\end{array}$} \\
\cline { 2 - 3 } & Array1[m] & Array2[m] & \\
\hline A & $(0,11)$ & $(0,-487)$ & \\
& $(0,0)$ & $(0,-500)$ & $(5000,400)$ \\
& $(2,-5)$ & $(3,-505)$ & \\
\hline B & $(0,9)$ & $(0,-789)$ & \\
& $(0,0)$ & $(0,-800)$ & $(5000,400)$ \\
& $(3,-6)$ & $(2,-805)$ & \\
\hline C & $(0,10)$ & $(0,-990)$ & \\
& $(0,0)$ & $(0,-1000)$ & $(5000,400)$ \\
& $(2,-4)$ & $(3,-1006)$ & \\
\hline
\end{tabular}

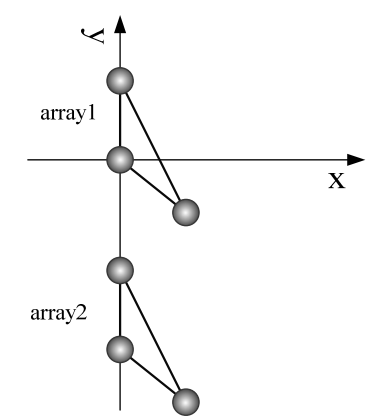

(a) Sensor topology 


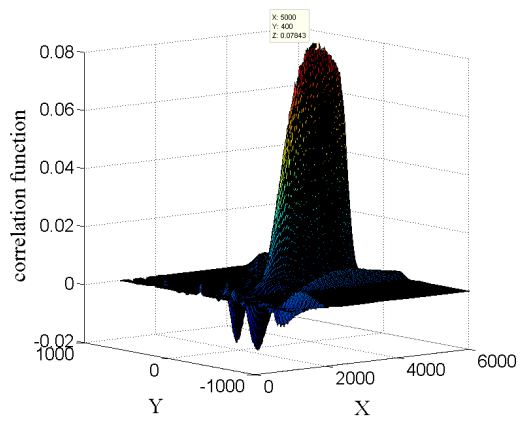

(b)The simulation result of the A array structure

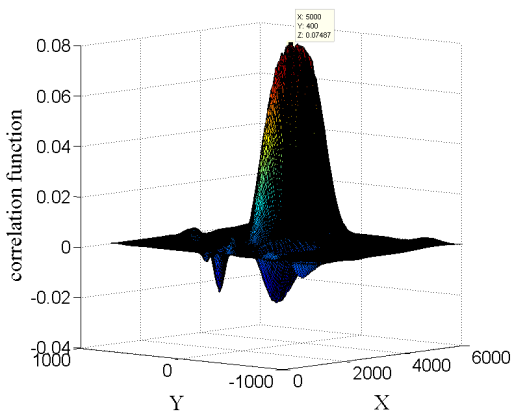

(c)The simulation result of the B array structure

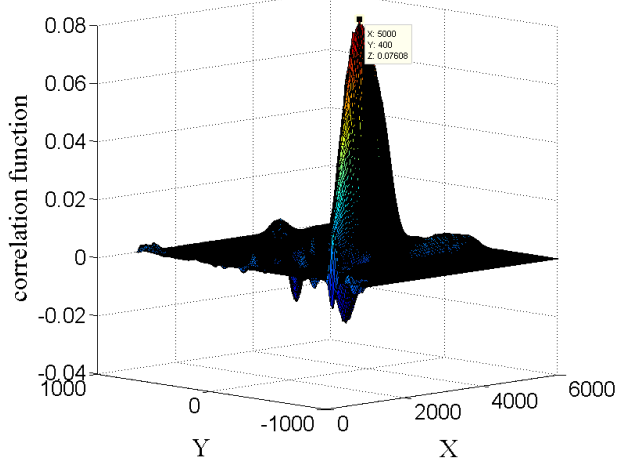

(d)The simulation result of the $\mathrm{C}$ array structure

Figure 2.Sensor topology and simulation results

As shown in Fig. 2(a)-(d), the simulations under the three array topologies A, B and C can accurately find the position of the sound source $(5000,400)$. In addition, it can be seen from the simulation results that changing the array spacing and inter-array spacing can accurately find the position of the sound source, so the limitation of sensor position in the array is not strict.

\subsection{SNR simulation results}

Simulation has been done under the conditions of $\mathrm{SNR}=1$ and $\mathrm{SNR}=0.04$. Fig. 3 shows the time domain signal when the SNR is 1 and 0.04, respectively. Fig. 4 shows simulation results of space location when the SNR is 1 and 0.04 , respectively.

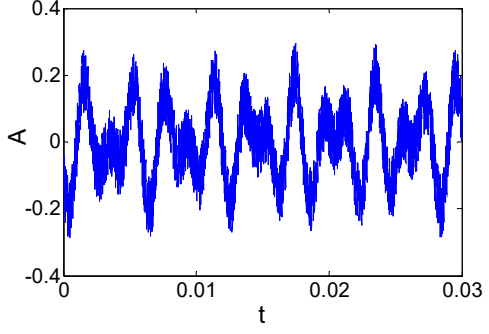

(a) Time-domain signal with a SNR of 1

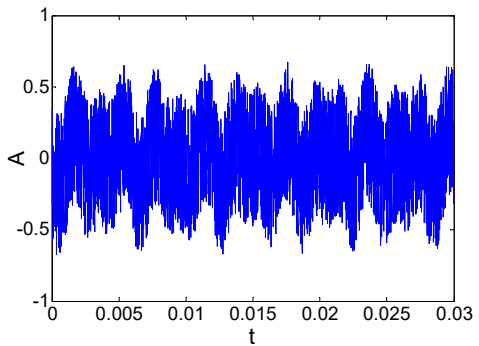

(b) Time-domain signal with a SNR of 1

Figure 3.Sensor array output time-domain analog signal

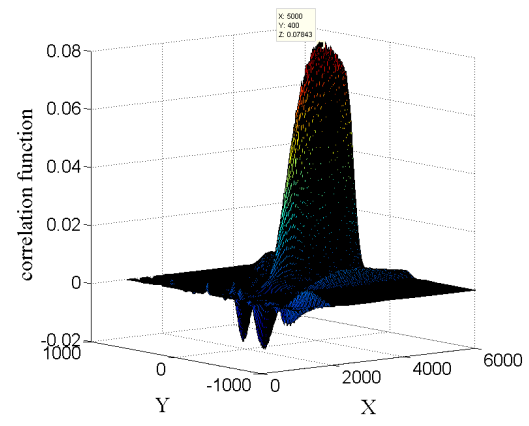

(a)Simulation result with SNR of 1

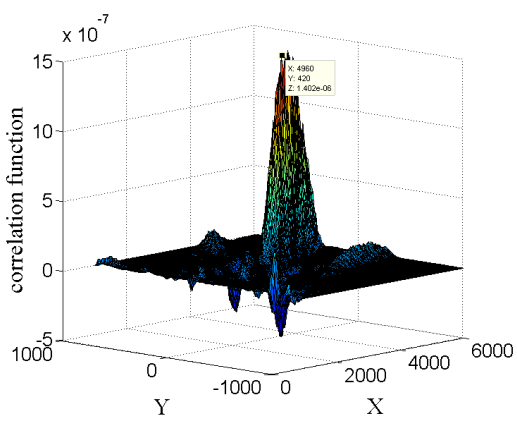

(b)Simulation result with SNR of 0.04

Figure 4. Space positioning simulation results

From Fig. 3 and Fig. 4, it can be seen that the source position $(5000,400)$ can be accurately found when the SNR is 1, which is consistent with the actual position. When SNR is 0.04 , the sound source location obtained by simulation is $(4960,425)$. Although there are errors in the results, it is basically tallies with the actual sound source position $(5000,400)$, in meters .

From the above simulation analysis, it can be seen that the sound source can be accurately positioned with six sensors (two arrays) in this method. In addition, the 
positioning method does not have high requirements for inter-array spacing spacing and array spacing. Thus, The sound field HBT interference principle can be used for spatial positioning, which can realize accurate positioning at long distance and low SNR.

\section{The positioning experiment in air}

Based on the principle and simulation of HBT interference location, the experiment of air localization is designed. The air experiment is shown in Fig. 5. The coordinate system as shown in Fig. 1 is established. The sound source $S$ is a continuous sound signal which is received by two microphone amplifiers arrays. STM32F103 was used for data collection. Finally, the collected data was calculated with matlab for correlation function, and the location of sound source was found.

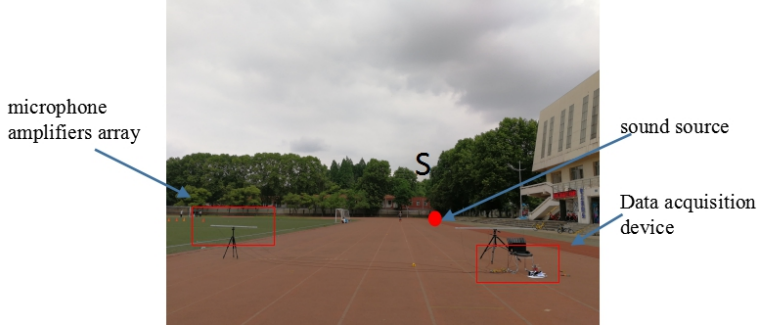

Figure 5. The air experiment

With the above method, the positioning test is carried out when the sound source at different distances. The sound sources at coordinates $(19.1,1.2)$ and $(42.0,1.2)$ are tested. The experimental results are shown in Fig.6(a)-(b). Considering the effects of microphone installation precision, environmental noise, wind speed and sound attenuation in the air, the final experimental results are consistent with the actual sound source position. The source position at 42 meters can be accurately located, and the positioning error is 0.1 meters.

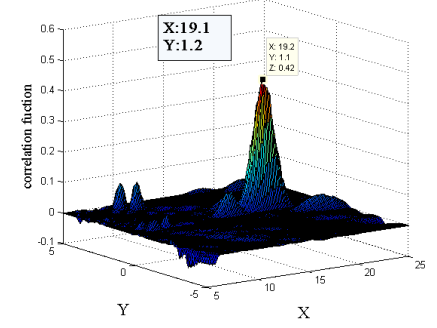

(a)Test results at 19 meters

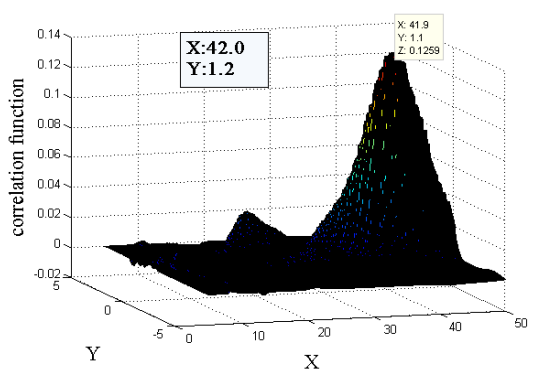

(b)Test results at 42 meters

Figure 6. Space positioning test results

\section{Conclusion}

A space location method based on the sound field HBT interference is presented. The source position can be obtained by analyzing the HBT correlation function of output signals. The simulation of the sound source localization is performed under different array topologies and SNRs. The results show that the precisely positioning of low SNR and long distance can be realized, and the sensor array topology does not require high. The location of sound source at different positions is realized through air experiment. This method provides a new idea to find relatively silent objects.

\section{Acknowledge}

This project is supported by National Natural Science Foundation of China (Grant No.51805154), State Key Laboratory of Precision Measuring Technology and Instruments (pilab1805) and Scientific Research Foundation for Doctoral Program of Hubei University of Technology (BSQD2017005).

\section{References}

1. Miao F, Yang D, Wen J, et al. Moving sound source localization based on triangulation method[J]. Journal of Sound \& Vibration, 2016, 385:93-103.

2. Yang Yu: Acoustic source localization research based on machine learning[D]Nanjing University of Posts and Telecommunications, 2017.

3. KE Wei, ZHANG Ming, ZHANG Tiecheng. Thr ee-dimensional sound source localization using $d$ istributed microphone arrays[J].Acta Acustica,201 7,42(03):361-369.

4. Potamitis I,Chen H,Tremoulis G: Tracking of multiple moving speakers with multiple microphone arrays(2004).

5. ZHANG Dawei, BAO Changchun, XIA Bingyin. Source localization based on time delay estimati on in complex environment $[\mathrm{J}]$ Journal on Comm unications, 2014(1):183-190.

6. Hmam H. Passive source localization from time of arrival measurements[C]// International Symposium on Wireless Pervasive Computing. IEEE, 2008:126129.

7. Chen W, Wang D, Wang W. Beamforming for information transfer in wireless sensor networks without perfect positioning[C]// Microwave Conf erence Proceedings, 2005. APMC 2005. Asia-Pa cific Conference Proceedings. IEEE, 2006:4 pp.

8. LIU Wuyun,YANG Wei.Design of sound source localization system based on microphone arrays [J].Piezoelectrics and Acoustooptics,2014,36(02):3 14-316. 